\title{
LOCAL HOMOGENEITY IN FUZZY TOPOLOGICAL SPACES
}

\author{
SAMER AL GHOUR
}

Received 11 May 2005; Revised 13 February 2006; Accepted 12 March 2006

Three types of local homogeneity in $L$-topological spaces are introduced and studied, and each is characterized and proved to be a good extension of local homogeneity in ordinary topological spaces. Many implications concerning them are introduced. The study deals with the $L$-topologically generated topological spaces.

Copyright (c) 2006 Hindawi Publishing Corporation. All rights reserved.

\section{Introduction}

Throughout this paper, $L$ is a fuzzy lattice, that is, a completely distributive lattice with an order-reversing involution ' on it, and with smallest element 0 and largest element 1 $(0 \neq 1)$. Let $X$ be a nonempty set. An $L$-set of $X$ is a function with domain $X$ and values in $L$; that is, an element of $L^{X} . L^{X}$ under the pointwise ordering:

$$
\begin{gathered}
\text { for } \lambda, \mu \in L^{X}, \quad \lambda \leq \mu \quad \text { in } L^{X} \text { iff } \lambda(x) \leq \mu(x) \text { in } L \forall x \in X, \\
\lambda^{\prime}(x)=(\lambda(x))^{\prime} \quad \forall x \in X
\end{gathered}
$$

is also a fuzzy lattice.

Throughout this paper, if $\left\{\lambda_{j}: j \in J\right\}$ is a collection of $L$-sets in $X$, then $\left(\vee \lambda_{j}\right)(x)=$ $\vee\left\{\lambda_{j}(x): j \in J\right\}, x \in X$; and $\left(\wedge \lambda_{j}\right)(x)=\wedge\left\{\lambda_{j}(x): j \in J\right\}, x \in X$. If $r \in L$, then $r_{X}$ denotes the fuzzy set given by $r_{X}(x)=r$ for all $x \in X$; that is, $r_{X}$ denotes the "constant" $L$-set of level $r$, that is, the smallest and the largest elements of $L^{X}$ are denoted, respectively, by $0_{X}$ and $1_{X}$. If $r_{X}$ is a constant $L$-set and $\lambda \in L^{X}$, then $r_{X} \wedge \lambda$ will be denoted by $r \lambda$. $\lambda \in L^{X}$ is called an $L$-crisp subset on $X$ if there exists an ordinary subset $A \subseteq X$ such that $\lambda=\chi_{A}: X \rightarrow\{0,1\} \subseteq L$, that is, if $\lambda$ is a characteristic function of some ordinary subset of $X$. An $L$-point on $X$ is an $L$-subset $x_{a} \in L^{X}$ defined as follows:

$$
\text { for every } y \in X, \quad x_{a}(y)= \begin{cases}a & \text { if } y=x \\ 0 & \text { if } y \neq x\end{cases}
$$

Hindawi Publishing Corporation International Journal of Mathematics and Mathematical Sciences Volume 2006, Article ID 81497, Pages 1-14

DOI 10.1155/IJMMS/2006/81497 
where $a \neq 0, x \in X$ is called the support of $x_{a}$ and $a$ the value (level) of $x_{a}$. Let $f: X \rightarrow Y$ be a function. We define

$$
f^{\rightarrow}: L^{X} \longrightarrow L^{Y}, \quad f^{-}: L^{Y} \longrightarrow L^{X}
$$

by

$$
f \rightarrow(\lambda)(y)= \begin{cases}\vee\left\{\lambda(x): x \in f^{-1}(\{y\})\right\} & \text { if } y \in \text { range } f \\ 0 & \text { if } y \notin \text { range } f\end{cases}
$$

and $f^{\leftarrow}(\mu)=\mu \circ f$.

An $L$-point in $X$ is said to belong to an $L$-set $\lambda$ in $X$ (notation: $x_{a} \in \lambda$ ) if and only if $a \leq \lambda(x)$.

An $L$-topological space is a pair $\left(L^{X}, \mathfrak{J}\right)$, where $X$ is a nonempty set, $L$ is a fuzzy lattice and $\mathfrak{I}$ called an $L$-topology on it is a subfamily of $L^{X}$ satisfying the following three axioms.

(1) $0_{X}, 1_{X} \in \mathfrak{J}$.

(2) If $\lambda, \mu \in \mathfrak{J}$, then $\lambda \wedge \mu \in \mathfrak{J}$.

(3) If $\left\{\lambda_{j}: j \in J\right\} \subseteq \mathfrak{J}$, then $\vee\left\{\lambda_{j}: j \in J\right\} \in \mathfrak{J}$.

The elements of $\mathfrak{I}$ are called open $L$-sets. An $L$-set $\mu$ is called closed if $\mu^{\prime} \in \mathfrak{J}$.

Let $(X, \tau)$ be an ordinary topological space, $L$ a complete lattice and $a \in L$. We define $\downarrow a=\{b \in L: b \leq a\}$ and we denote the cotopology on $L$ generated by the subbase $\{\downarrow a$ : $a \in L\}$ by $\underline{\Omega}(L)$. The correspondent topology of $\underline{\Omega}(L)$ is denoted by $\Omega(L)$. A mapping $f$ : $X \rightarrow L$ is called lower semicontinuous if and only if $f:(X, \tau) \rightarrow(L, \Omega(L))$ is continuous. The class of all lower semicontinuous mappings from $X$ to $L$ is an $L$-topology on $X$. This $L$-topology is denoted by $\omega_{L}(\tau)$.

If $\left(L^{X}, \mathfrak{J}\right)$ is an $L$-topological space and $a \in L$, then the set $\left\{\lambda^{-1}(\downarrow a): \lambda \in \mathfrak{J}\right\}$ is a cotopology on $X$. The correspondent topology of $\left\{\lambda^{-1}(\downarrow a): \lambda \in \mathfrak{J}\right\}$ is denoted by $\mathfrak{J}_{a}$.

Let $\left(L^{X}, \mathfrak{I}_{1}\right)$ and $\left(L^{Y}, \mathfrak{J}_{2}\right)$ be two $L$-topological spaces. A mapping $f:\left(L^{X}, \mathfrak{J}_{1}\right) \rightarrow\left(L^{Y}, \mathfrak{I}_{2}\right)$ will be called $L$-continuous if and only if for every $\lambda \in \mathfrak{I}_{2}, f^{-}(\lambda) \in \mathfrak{I}_{1}$. A bijective map $h$ : $\left(L^{X}, \mathfrak{I}_{1}\right) \rightarrow\left(L^{Y}, \mathfrak{I}_{2}\right)$ will be called $L$-homeomorphism if and only if $h$ and $h^{-1}$ are both $L$ continuous. Throughout this paper, we will denote the group of all $L$-homeomorphisms from the $L$-topological space $\left(L^{X}, \mathfrak{J}\right)$ onto itself by $\operatorname{LHOM}\left(L^{X}, \mathfrak{I}\right)$.

Definition 1.1 [10]. Associated with a given $L$-topological space $\left(L^{X}, \mathfrak{J}\right)$ and arbitrary nonempty ordinary subset $M$ of $X$, define the induced $L$-topology on $M$ or the relative $L$-topology on $M$ by

$$
\mathfrak{J}_{M}=\left\{\lambda_{\mid M}: \lambda \in \mathfrak{J}\right\}
$$

The corresponding pair $\left(L^{M}, \mathfrak{J}_{M}\right)$ is called an open (a closed) subspace if and only if the $L$-crisp subset $\chi_{M}$ is open (closed) in $\left(L^{X}, \mathfrak{I}\right)$.

Definition 1.2 [11]. For a property $\mathbf{P}$ in ordinary topological spaces, a property $\mathbf{P}^{*}$ of $L$ topological spaces is called a good extension of $\mathbf{P}$, if for every ordinary topological space $(X, \tau),(X, \tau)$ has $\mathbf{P}$ if and only if $\left(L^{X}, \omega_{L}(\tau)\right)$ has $\mathbf{P}^{*}$. 
Throughout this paper the group of all homeomorphisms from the topological space $(X, \tau)$ onto itself will be denoted by $\operatorname{HOM}(X, \tau)$.

Definition 1.3 [12]. A topological space $(X, \tau)$ is called homogeneous if for any two points $x, y \in X$ there exists $h \in \operatorname{HOM}(X, \tau)$ such that $h(x)=y$.

Definition $1.4[5]$. Let $(X, \tau)$ be a topological space and let $\tilde{\tau}$ be the equivalence relation on $X$ defined by $x \tilde{\tau} y$ if there exists $h \in \operatorname{HOM}(X, \tau)$ such that $h(x)=y$. Then the equivalence class $C_{x}^{\tau}=\{y \in X: x \tilde{\tau} y\}$ is called the homogeneous component of $(X, \tau)$ determined by $x$.

Definition 1.5 [8]. A topological space $(X, \tau)$ is SLH (strongly locally homogeneous) if $\tau$ has a base $\boldsymbol{\beta}$ such that for any nonempty $U \in \boldsymbol{\beta}$ and for any $x, y \in U$, there exists $h \in$ $\operatorname{HOM}(X, \tau)$ such that $h(x)=y$ and $h(t)=t$ for all $t \notin U$.

Definition 1.6 [6]. A topological space $(X, \tau)$ is called LH (locally homogeneous) at $x$ in $X$ provided that there exists an open set $U$ in $X$ containing $x$ such that for any $y \in U$ there is $h \in \operatorname{HOM}(X, \tau)$ such that $h(x)=y$. A topological space $(X, \tau)$ is called LH if it is LH at each $x \in X$.

From now on $I$ will denote the complete distributive lattice $[0,1]$ with the usual order and order-reversing involution ',$r^{\prime}=1-r$ for every $r \in I$.

Definition 1.7. An $L$-topological space $\left(L^{X}, \mathfrak{J}\right)$ is called $L$-homogeneous if for any two points $x, y \in X$, there exists $h \in \operatorname{LHOM}\left(L^{X}, \mathfrak{J}\right)$ such that $h(x)=y$.

Definition 1.7 generalized [7, Definition 2.1] for $n=1$ in which the authors called $I$ homogeneous $I$-topological, a homogeneous fuzzy topological space.

Definition 1.8. Let $\left(L^{X}, \mathfrak{J}\right)$ be an $L$-topological space and let $\widetilde{\mathfrak{J}}$ be the equivalence relation on $X$ defined by $x \widetilde{\mathfrak{I}} y$ if there exists $h \in \operatorname{LHOM}\left(L^{X}, \mathfrak{I}\right)$ such that $h(x)=y$. Then the equivalence class $L-C_{x}^{\mathfrak{J}}=\{y \in X: x \widetilde{\mathfrak{I}} y\}$ is called the $L$-homogeneous component of $\left(L^{X}, \mathfrak{I}\right)$ determined by $x$.

Definition 1.9 [11]. Let $\left(L^{X}, \mathfrak{J}\right)$ be an $L$-topological space. A family $\boldsymbol{\beta}$ of open $L$-sets is called a base for $\mathfrak{J}$ if each nonzero member of $\mathfrak{I}$ can be written as a join of members of $\boldsymbol{\beta}$.

Throughout this paper, if $\lambda$ is a fuzzy set in $X$, then the support of $\lambda$ is denoted by $S(\lambda)$ and defined by $S(\lambda)=\lambda^{-1}(L-\{0\})$.

Definition 1.10. Let $\left(L^{X}, \mathfrak{J}\right)$ be an $L$-topological space and let $\boldsymbol{\beta}$ be a base for $\mathfrak{J}$. Then $\boldsymbol{\beta}$ is a representable base for $\mathfrak{I}$ if for any nonzero $\lambda \in \beta$ and for any $x, y \in S(\lambda)$, there exists $h \in \operatorname{FHOM}\left(L^{X}, \mathfrak{J}\right)$ such that $h(x)=y$ and $h(t)=t$ for all $t \notin S(\lambda)$.

Definition 1.11. An $L$-topological space $\left(L^{X}, \mathfrak{J}\right)$ is said to be $L$-SLH ( $L$-strongly locally homogeneous) if $\mathfrak{I}$ has a representable base.

Definitions 1.8, 1.9, and 1.11 generalize [3, Definitions 2.1, 3.1, and 3.2], respectively. In fact, the fuzzy lattice $I$ is replaced by arbitrary fuzzy lattice $L$.

Since Zadeh [13] introduced the fundamental concept of fuzzy set (I-set) and Chang [4] defined $I$-topological spaces, several mathematicians extended the main notions of 
general topology to fuzzy set theory, such as the separation and countability axioms, compactness, connectedness, paracompactness, metric space, and so forth. Recently some homogeneity types were extended to include $I$-topological spaces. In [1], the author extended homogeneity, $n$-homogeneity, weakly $n$-homogeneity, countable dense homogeneity, dense homogeneity, and strong local homogeneity as known ordinary topological concepts to include $I$-topological spaces. Results concerning homogeneity, $n$-homogeneity and weakly $n$-homogeneity appeared in [7], in which the authors introduced some open questions; then, in [2], Al Ghour had solved two of them. The results in [1] concerning $I$-homogeneous components and $I$-strong local homogeneity appeared in [3]. In [9], the author defined $L$-sets as a generalization of $I$-sets and then used $L$-sets to define $L$-topological spaces as a generalization of $I$-topological spaces. After that, many ordinary topological concepts extended in $L$-topological spaces. As a generalization of both homogeneity and strong local homogeneity in ordinary topological spaces, local homogeneity was recently introduced in [6]. In the present paper, three types of local homogeneity in $L$-topological spaces will be introduced and studied, each of which will be characterized and proved to be a good extension of local homogeneity in ordinary topological spaces. Many implications concerning them will be studied. The study will deal with the $L$-topologically generated topological spaces. Many counter examples relevant to the relations obtained in this paper will be given.

Throughout this paper, for any nonempty set $X, \tau_{\text {disc }}$ will denote the discrete topology on $X$.

The following five propositions will generalize [3, Theorems 2.4, 2.8, 3.10, 2.6, 3.6] respectively, to include $L$-topological spaces instead of $I$-topological spaces. These propositions will be used in the sequel.

Proposition 1.12. If $\left(L^{X}, \mathfrak{J}\right)$ is an $L$-topological space and $L-C_{x}^{\mathfrak{J}}$ is an $L$-homogeneous component of $\left(L^{X}, \mathfrak{I}\right)$, then for any $h \in \operatorname{LHOM}\left(L^{X}, \mathfrak{I}\right), h\left(L-C_{x}^{\mathfrak{I}}\right)=L-C_{x}^{\mathfrak{I}}$.

Proof. The proof is similar to that used in [3, Theorem 2.4].

The following lemma will be used in the sequel.

Lemma 1.13. Let $\left(X, \tau_{1}\right)$ and $\left(Y, \tau_{2}\right)$ be two topological spaces and let $L$ be a fuzzy lattice. Then $f:\left(X, \tau_{1}\right) \rightarrow\left(Y, \tau_{2}\right)$ is continuous if and only if $f:\left(L^{X}, \omega_{L}\left(\tau_{1}\right)\right) \rightarrow\left(L^{Y}, \omega_{L}\left(\tau_{2}\right)\right)$ is $L$ continuous.

Proof. Follows from [10, Corollary 7.2.5, page 370].

Proposition 1.14. Let $(X, \tau)$ be a topological space and let $L$ be a fuzzy lattice. Then $C_{x}^{\tau}=$ $L-C_{x}^{\omega_{L}(\tau)}$ for all $x \in X$.

Proof. Lemma 1.13.

Proposition 1.15. If $\left(L^{X}, \mathfrak{I}\right)$ is an $L$-SLH L-topological space, then every L-homogeneous component $L-C_{x}^{\mathfrak{J}}$ of $\left(L^{X}, \mathfrak{J}\right)$ is clopen in $\left(X, \mathfrak{J}_{0}\right)$.

Proof. It is sufficient to prove $L-C_{x}^{\mathfrak{J}} \in \mathfrak{J}_{0}$ for every $x \in X$. Let $x \in X$ and let $y \in L-C_{x}^{\mathfrak{J}}$, then there exists $f \in \operatorname{LHOM}\left(L^{X}, \mathfrak{J}\right)$ such that $f(y)=x$. Choose a representable base $\beta$ for $\mathfrak{I}$. Choose $\lambda \in \boldsymbol{\beta}$ such that the $L$-point has support $y$ and level $1, y_{1} \in \lambda$. Then $y \in S(\lambda)$. 
Let $t \in S(\lambda)$, then there exists $g \in \operatorname{LHOM}\left(L^{X}, \mathfrak{J}\right)$ such that $g(t)=y$ and $g(b)=b$ for all $b \notin S(\lambda)$. Therefore, $f \circ g \in \operatorname{LHOM}\left(L^{X}, \mathfrak{J}\right)$ with $(f \circ g)(t)=x$ and hence $t \in L-C_{x}^{\mathfrak{J}}$. So $S(\lambda) \in \mathfrak{I}_{0}$ and $S(\lambda) \subseteq L-C_{x}^{\mathfrak{J}}$. Thus $L-C_{x}^{\mathfrak{J}} \in \mathfrak{J}_{0}$.

The following lemma will be used in proving the next proposition.

Lemma 1.16. Let $\left(L^{X}, \mathfrak{I}\right)$ and $\left(L^{Y}, S\right)$ be two L-topological spaces, and let $f:\left(L^{X}, \mathfrak{I}\right) \rightarrow$ $\left(L^{Y}, S\right)$ be an L-continuous mapping. Then $f:\left(L^{X}, \mathfrak{J}_{a}\right) \rightarrow\left(L^{Y}, S_{a}\right)$ is continuous for all $a \in$ $L-\{1\}$.

Proof. Let $a \in L-\{1\}$ and let $\lambda \in S$, then $f^{-1}\left(\lambda^{-1}(\downarrow a)\right)=(\lambda \circ f)^{-1}(\downarrow a)=\left(f^{\leftarrow}(\lambda)\right)^{-1}(\downarrow$ a). Since $f:\left(L^{X}, \mathfrak{J}\right) \rightarrow\left(L^{Y}, S\right)$ is $L$-continuous, then $f^{-}(\lambda) \in \mathfrak{I}$ and so $\left(f^{-}(\lambda)\right)^{-1}(\downarrow a)$ is closed.

According to Lemma 1.16, the following result follows easily.

Proposition 1.17. Let $\left(L^{X}, \mathfrak{J}\right)$ be an L-topological space and let $a \in L-\{1\}$. Then $C_{x}^{\mathfrak{I}_{a}}=$ $\bigcup_{y \in C_{x}^{\mathfrak{j} a}} L-C_{y}^{\mathfrak{J}}$.

The following lemma will be used in the sequel.

Lemma 1.18. Let $X$ be a nonempty set and let $\left\{\lambda_{\alpha}: \alpha \in \Lambda\right.$ \} be a family of L-subsets of $X$. Then $\bigcup_{\alpha \in \Lambda} S\left(\lambda_{\alpha}\right)=S\left(\bigvee_{\alpha \in \Lambda} \lambda_{\alpha}\right)$.

Proof. Let $x \in \bigcup_{\alpha \in \Lambda} S\left(\lambda_{\alpha}\right)$. Then there exists $\alpha_{\circ} \in \Lambda$ such that $x \in S\left(\lambda_{\alpha_{\circ}}\right)$. Therefore, $\left(\bigvee_{\alpha \in \Lambda} \lambda_{\alpha}\right)(x)=\sup \left\{\lambda_{\alpha}(x): \alpha \in \Lambda\right\} \geq \lambda_{\alpha_{\circ}}(x)>0$ and hence $x \in S\left(\bigvee_{\alpha \in \Lambda} \lambda_{\alpha}\right)$. On the other hand, if $x \in S\left(\bigvee_{\alpha \in \Lambda} \lambda_{\alpha}\right)$, then $\sup \left\{\lambda_{\alpha}(x): \alpha \in \Lambda\right\}>0$ and so there exists $\alpha_{\circ} \in \Lambda$ such that $\lambda_{\alpha_{\circ}}(x)>0$. Therefore, $x \in S\left(\lambda_{\alpha_{\circ}}\right)$ and hence $x \in \bigcup_{\alpha \in \Lambda} S\left(\lambda_{\alpha}\right)$.

The following lemma will be used in proving the next proposition.

Lemma 1.19. Let $(X, \tau)$ be a topological space and let $L$ be a fuzzy lattice. Then

(1) if $\beta$ is a base for $\tau$, then $\beta^{*}=\left\{\lambda \in \omega_{L}(\tau): S(\lambda) \in \beta\right\}$ is a base for $\omega_{L}(\tau)$;

(2) if $\digamma$ is a base for $\omega_{L}(\tau)$, then $\digamma^{*}=\{S(\lambda): \lambda \in \digamma\}$ is a base for $\tau$.

Proof. (1) Let $\lambda \in \omega_{L}(\tau)$ with $\lambda \neq 0_{X}$. Then $S(\lambda) \in \tau$ with $S(\lambda) \neq \varnothing$ and so there exists $\left\{B_{\alpha}: \alpha \in \Lambda\right\} \subseteq \beta$ such that $S(\lambda)=\bigcup\left\{B_{\alpha}: \alpha \in \Lambda\right\}$. For each $\alpha \in \Lambda, \lambda \wedge \chi_{B_{\alpha}} \in \omega_{L}(\tau)$ with $S\left(\lambda \wedge \chi_{B_{\alpha}}\right)=B_{\alpha} \in \beta$ and so $\lambda \wedge \chi_{B_{\alpha}} \in \beta^{*}$. Since $\lambda=\bigvee\left\{\lambda \wedge \chi_{B_{\alpha}}: \alpha \in \Lambda\right\}$, the proof is complete.

(2) Let $U \in \tau$ with $U \neq \varnothing$. Then $\chi_{U} \in \omega_{L}(\tau)$ with $\chi_{U} \neq 0_{X}$ and so there exists $\left\{\lambda_{\alpha}\right.$ : $\alpha \in \Lambda\} \subseteq \digamma$ such that $\chi_{U}=\bigvee\left\{\lambda_{\alpha}: \alpha \in \Lambda\right\}$. Therefore, by Lemma 1.18 it follows that $U=$ $S\left(\chi_{U}\right)=\bigcup\left\{S\left(\lambda_{\alpha}\right): \alpha \in \Lambda\right\}$.

Proposition 1.20. The L-SLH property in L-topological spaces is a "good extension" of SLH property in ordinary topological spaces.

Proof. Let $(X, \tau)$ be a topological space and let $L$ be a fuzzy lattice. Suppose $(X, \tau)$ is SLH. Then $\tau$ has a representable base $\boldsymbol{\beta}$. So by Lemma $1.19(1), \boldsymbol{\beta}^{*}=\left\{\lambda \in \omega_{L}(\tau): S(\lambda) \in \boldsymbol{\beta}\right\}$ is a base for $\omega_{L}(\tau)$. Let $\lambda \in \beta^{*}$ with $\lambda \neq 0_{X}$ and let $x, y \in S(\lambda)$. Then $S(\lambda) \in \boldsymbol{\beta}$ with $S(\lambda) \neq \varnothing$. Therefore, there exists $h \in \operatorname{HOM}(X, \tau)$ such that $h(x)=y$. Lemma 1.13 completes the 
proof that $\left(L^{X}, \omega_{L}(\tau)\right)$ is $L$-SLH. The proof of the other direction is similar to the above one.

The following proposition will generalize [7, Theorem 2.9] for the case $n=1$.

Proposition 1.21. The homogeneity property in L-topological spaces is a "good extension" of homogeneity property in ordinary topological spaces.

Proof. Lemma 1.13.

Finally, we selected four results from [6], those results will be used in the sequel.

Proposition 1.22 [6]. A topological space $(X, \tau)$ is LH at $x$ if and only if $C_{x}^{\tau}$ is open.

Proposition 1.23 [6]. In ordinary spaces local homogeneity at some point does not imply local homogeneity.

Proposition 1.24 [6]. Every SLH topological space is LH but not conversely.

Proposition 1.25 [6]. Every homogeneous topological space is LH but not conversely.

\section{Locally homogeneous $L$-topological spaces}

Let us begin by the following definition.

Definition 2.1. An $L$-topological space $\left(L^{X}, \mathfrak{J}\right)$ is called $L$-LH ( $L$-locally homogeneous) at $x$ in $X$ provided that there exists an open $L$-set $\lambda$ in $X$ such that $x \in S(\lambda)$ and for any $y \in S(\lambda)$ there is $h \in \operatorname{LHOM}\left(L^{X}, \mathfrak{J}\right)$ such that $h(x)=y$. An $L$-topological space $\left(L^{X}, \mathfrak{J}\right)$ is called $L$-LH if it is $L-\mathrm{LH}$ at each $x \in X$.

The following lemma will be used in the proof of the next main result.

Lemma 2.2. Let $h: X \rightarrow Y$ be a bijective function and let $\lambda$ be an $L$-subset of $X$. Then $h(S(\lambda))$ $=S(h \rightarrow(\lambda))$.

Proof. Let $y \in h(S(\lambda))$. Then there exists $x \in S(\lambda)$ such that $h(x)=y$. Now $\left(h^{\rightarrow}(\lambda)\right)(y)=$ $\lambda\left(h^{-1}(y)\right)=\lambda(x)>0$. Therefore, $y \in S\left(h^{\rightarrow}(\lambda)\right)$. Conversely, if $y \in S\left(h^{\rightarrow}(\lambda)\right)$, then $\left(h^{\rightarrow}(\lambda)\right)(y)$ $>0$ and so $\lambda\left(h^{-1}(y)\right)>0$. Let $x=h^{-1}(y)$. Then $x \in S(\lambda)$ and $h(x)=y$. Therefore, $y \in$ $h(S(\lambda))$.

Theorem 2.3. Let $\left(L^{X}, \mathfrak{J}\right)$ be an $L$-topological space and let $x \in X$. Then the following are equivalent.

(1) $\left(L^{X}, \mathfrak{I}\right)$ is $L-L H$ at $x$.

(2) There exists an open $L$-set $\lambda$ in $X$ such that $x \in S(\lambda) \subseteq L-C_{x}^{\mathfrak{J}}$.

(3) $\left(L^{X}, \mathfrak{J}\right)$ is $L-L H$ at each point $y \in L-C_{x}^{\mathfrak{I}}$.

(4) There exists an open $L$-set $\lambda$ in $X$ such that $S(\lambda)=L-C_{x}^{\mathfrak{J}}$.

(5) There exists $r_{x} \in L-\{0\}$ such that $\bigvee\left\{\lambda \in \mathfrak{I}: S(\lambda) \subseteq L-C_{x}^{\mathfrak{I}}\right\}=r_{x} \chi_{L-C_{x}^{\mathfrak{J}}}$.

(6) There exists $r_{x} \in L-\{0\}$ such that $r_{x} \chi_{L-C_{x}^{\mathfrak{J}}} \in \mathfrak{J}$.

(7) $L-C_{x}^{\mathfrak{J}} \in \mathfrak{J}_{0}$. 
Proof. (1) $\Rightarrow(2)$. By (1) there exists an open $L$-set $\lambda$ in $X$ such that $x \in S(\lambda)$ and for any $y \in S(\lambda)$ there is $h \in \operatorname{LHOM}\left(L^{X}, \mathfrak{J}\right)$ such that $h(x)=y$. Therefore, $x \in S(\lambda) \subseteq L-C_{x}^{\mathfrak{J}}$.

$(2) \Rightarrow(3)$. Let $y \in L-C_{x}^{\mathfrak{J}}$. Then there exists $h \in \operatorname{LHOM}\left(L^{X}, \mathfrak{J}\right)$ such that $h(x)=y$. By (2), there exists an open $L$-set $\lambda$ in $X$ such that $x \in S(\lambda) \subseteq L-C_{x}^{\mathfrak{J}}$ and so $y \in h(S(\lambda)) \subseteq$ $h\left(L-C_{x}^{\mathfrak{I}}\right)$. Thus, by Proposition 1.12 and Lemma 2.2, it follows that $y \in S\left(h^{\rightarrow}(\lambda)\right) \subseteq L-$ $C_{x}^{\mathfrak{I}}=L-C_{y}^{\mathfrak{J}}$. This completes the proof of this implication.

(3) $\Rightarrow(4)$. By (3), for each $y \in L-C_{x}^{\mathfrak{J}}$, there exists $\lambda_{y} \in \mathfrak{J}$ such that $y \in S\left(\lambda_{y}\right) \subseteq L-$ $C_{y}^{\mathfrak{J}}=L-C_{x}^{\mathfrak{J}}$. Therefore, $L-C_{x}^{\mathfrak{I}}=\bigcup_{y \in L-C_{x}^{\mathfrak{I}}} S\left(\lambda_{y}\right)$. Let $\lambda=\bigvee_{y \in L-C_{x}^{\mathfrak{J}}} \lambda_{y}$. Then $\lambda \in \mathfrak{J}$ and by Lemma 1.18, it follows that $S(\lambda)=L-C_{x}^{\mathfrak{J}}$.

(4) $\Rightarrow(5)$. Let $\mu=\bigvee\left\{\lambda \in \mathfrak{I}: S(\lambda) \subseteq L-C_{x}^{\mathfrak{J}}\right\}$. Then $\mu \in \mathfrak{I}$ and by (4) and Lemma 1.18, it follows that $S(\mu)=L-C_{x}^{\mathfrak{J}}$. Let $r_{x}=\mu(x)$. Then $r_{x}>0$. We are going to show that $\mu=$ $r_{x} \chi_{C_{x}^{\mathfrak{g}}}$. Since $S(\mu)=L-C_{x}^{\mathfrak{J}}, \mu_{\mid X-L-C_{x}^{\mathfrak{j}}}=0_{X-L-C_{x}^{\mathfrak{j}}}$. On the other hand, let $y \in L-C_{x}^{\mathfrak{J}}$. Then there exists $h \in \operatorname{LHOM}\left(L^{X}, \mathfrak{I}\right)$ such that $h(x)=y$. Since $S(\mu)=L-C_{x}^{\mathfrak{I}}$, then $h(S(\mu))=$ $h\left(L-C_{x}^{\mathfrak{J}}\right)$ and $h^{-1}(S(\mu))=h^{-1}\left(L-C_{x}^{\mathfrak{J}}\right)$. So by Proposition 1.12 and Lemma 2.2, it follows that $S\left(h^{\rightarrow}(\mu)\right)=S\left(h^{\leftarrow}(\mu)\right)=L-C_{x}^{\mathfrak{J}}$. Therefore, by the definition of $\mu$, it follows that $h^{\rightarrow}(\mu) \leq \mu$ and $h^{\leftarrow}(\mu) \leq \mu$. Hence $\left(h^{\rightarrow}(\mu)\right)(y) \leq \mu(y)$ and $\left(h^{-}(\mu)\right)(x) \leq \mu(x)$. Thus $\mu(x) \leq \mu(y)$ and $\mu(y) \leq \mu(x)$ and so $\mu(y)=\mu(x)=r_{x}$. This completes the proof that $\mu=r_{x} \chi_{L-C_{x}^{\mathfrak{g}}}$.

$(5) \Rightarrow(6)$ and $(6) \Rightarrow(7)$ are obvious.

(7) $\Rightarrow(1)$. If $L-C_{x}^{\mathfrak{J}} \in \mathfrak{J}_{0}$, then there exists $\lambda \in \mathfrak{J}$ such that $\lambda^{-1}(L-\{0\})=L-C_{x}^{\mathfrak{J}}$ and so $S(\lambda)=L-C_{x}^{\mathfrak{J}}$. Therefore, $x \in S(\lambda)$ and for each $y \in S(\lambda)$, there exists $h \in \operatorname{LHOM}\left(L^{X}\right.$, $\mathfrak{J})$ such that $h(x)=y$. Hence, $\left(L^{X}, \mathfrak{J}\right)$ is $L-\mathrm{LH}$ at $x$.

Corollary 2.4. Let $\left(L^{X}, \mathfrak{J}\right)$ be an $L$-topological space. Then the following are equivalent.

(1) $\left(L^{X}, \mathfrak{I}\right)$ is $L-L H$.

(2) For each $x \in X$, there exists an open $L$-set $\lambda$ in $X$ such that $x \in S(\lambda) \subseteq L-C_{x}^{\mathfrak{J}}$.

(3) For each $x \in X$, there exists an open $L$-set $\lambda$ in $X$ such that $S(\lambda)=L-C_{x}^{\mathfrak{I}}$.

(4) For each $x \in X$, there exists $r_{x} \in L-\{0\}$ such that $\bigvee\left\{\lambda \in \mathfrak{J}: S(\lambda) \subseteq L-C_{x}^{\mathfrak{J}}\right\}=$ $r_{x} \chi_{L-C_{x}^{5}}$.

(5) For each $x \in X$, there exists $r_{x} \in L-\{0\}$ such that $r_{x} \chi_{L-C_{x}^{\mathfrak{g}}} \in \mathfrak{J}$.

(6) The L-homogeneous components of $\left(L^{X}, \mathfrak{I}\right)$ are clopen in $\left(X, \mathfrak{I}_{0}\right)$.

The following result shows that $L$-local homogeneity at some point in $L$-topological spaces is a "good extension" of the local homogeneity at some point in ordinary topological spaces.

Theorem 2.5. Let $(X, \tau)$ be a topological space, $x \in X$, and $L$ a fuzzy lattice. Then $(X, \tau)$ is $L H$ at $x$ if and only if $\left(L^{X}, \omega_{L}(\tau)\right)$ is L-LH at $x$.

Proof. Suppose that $(X, \tau)$ is $L-\mathrm{LH}$ at $x$. Then by Proposition 1.22, it follows that $C_{x}^{\tau} \in \tau$. Let $\lambda=\chi_{C_{x}^{\tau}}$. Then $\lambda \in \omega_{L}(\tau)$ and $S(\lambda)=C_{x}^{\tau}$. Therefore, by Proposition 1.14, it follows that $S(\lambda)=L-C_{x}^{\omega_{L}(\tau)}$. Hence by part (4) of Theorem 2.3, it follows that $\left(L^{X}, \omega_{L}(\tau)\right)$ is $L$-LH at $x$. Conversely, if $\left(L^{X}, \omega_{L}(\tau)\right)$ is $L$-LH at $x$, then by part (4) of Theorem 2.3 and Proposition 1.14, it follows that there exists $\lambda \in \omega_{L}(\tau)$ such that $S(\lambda)=C_{x}^{\tau}$. Now by the definition of $\omega_{L}(\tau)$ we must have $\lambda^{-1}(\downarrow 0)=S(\lambda) \in \tau$. Therefore, by Proposition 1.22, it follows that $(X, \tau)$ is LH at $x$. 
Corollary 2.6. The L-LH property in L-topological spaces is a "good extension" of the LH property in ordinary topological spaces.

The following result follows directly from Proposition 1.23 and Theorem 2.5.

Corollary 2.7. In L-topological spaces, L-local homogeneity at some point does not imply L-local homogeneity.

The following two results show that $L$-local homogeneity in $L$-topological spaces generalizes both $L$-homogeneity and $L$-strong local homogeneity.

Theorem 2.8. Every L-SLH L-topological space is a $L-L H$.

Proof. Follows by Proposition 1.15 and part (6) of Corollary 2.4.

Theorem 2.9. Every L-homogeneous L-topological space is an $L-L H$.

Proof. If $\left(L^{X}, \mathfrak{J}\right)$ is an $L$-homogeneous $L$-topological space, then for each $x \in X, S\left(1_{X}\right)=$ $X=L-C_{x}^{\mathfrak{I}}$. Therefore, by part (3) of Corollary 2.4 , it follows that $\left(L^{X}, \mathfrak{J}\right)$ is $L$-LH.

Theorem 2.10. If $\left(L^{X}, \mathfrak{I}\right)$ is an $L$-LH L-topological space and $\left(X, \mathfrak{I}_{0}\right)$ is connected, then $\left(L^{X}, \mathfrak{J}\right)$ is L-homogeneous.

Proof. Choose $x \in X$. Since $\left(L^{X}, \mathfrak{J}\right)$ is an $L$-LH $L$-topological space, then by part (6) of Corollary 2.4, it follows that $L-C_{x}^{\mathfrak{J}}$ is a clopen in the connected topological space $\left(X, \mathfrak{J}_{0}\right)$. This means that $L-C_{x}^{\mathfrak{J}}=X$, hence $\left(L^{X}, \mathfrak{J}\right)$ is an $L$-homogeneous.

Corollary 2.11. If $\left(L^{X}, \mathfrak{I}\right)$ is an $L$-SLH L-topological space and $\left(X, \mathfrak{I}_{0}\right)$ is connected, then $\left(L^{X}, \mathfrak{J}\right)$ is L-homogeneous.

Corollary 2.12 [3]. If $\left(I^{X}, \mathfrak{J}\right)$ is an I-SLH I-topological space and $\left(I^{X}, \mathfrak{J}\right)$ is connected, then $\left(I^{X}, \mathfrak{J}\right)$ is I-homogeneous.

\section{Magnitude and $L$-local homogeneity in $L$-topological spaces}

From Theorem 2.3, in a natural way we define the following.

Definition 3.1. Let $\left(L^{X}, \mathfrak{J}\right)$ be an $L$-LH $L$-topological space at $x$ and let

$$
\bigvee\left\{\lambda \in \mathfrak{J}: S(\lambda) \subseteq L-C_{x}^{\mathfrak{I}}\right\}=r_{x} \chi_{C_{x}^{\mathfrak{g}}}
$$

Then $r_{x}$ is called the magnitude of $\left(L^{X}, \mathfrak{J}\right)$ at $x$.

Proposition 3.2. Let $\left(L^{X}, \mathfrak{J}\right)$ be an $L$-LH L-topological space at $x$ and let $r_{x}$ be the magnitude of $\left(L^{X}, \mathfrak{J}\right)$ at $x$. Then the magnitude of $\left(L^{X}, \mathfrak{J}\right)$ at each $y \in L-C_{x}^{\mathfrak{J}}$ equals to $r_{x}$.

Proof. Let $y \in L-C_{x}^{\mathfrak{J}}$. Denote the magnitude of $\left(L^{X}, \mathfrak{J}\right)$ at $y$ by $r_{y}$. Since $y \in L-C_{x}^{\mathfrak{J}}$, $L-C_{x}^{\mathfrak{J}}=L-C_{y}^{\mathfrak{J}}$ and so

$$
\bigvee\left\{\lambda \in \mathfrak{J}: S(\lambda) \subseteq L-C_{x}^{\mathfrak{I}}\right\}=\bigvee\left\{\lambda \in \mathfrak{J}: S(\lambda) \subseteq L-C_{y}^{\mathfrak{I}}\right\}
$$

Therefore, $r_{x} \chi_{L-C_{x}^{\mathfrak{s}}}=r_{y} \chi_{L-C_{y}^{\mathfrak{y}}}=r_{y} \chi_{L-C_{x}^{\mathfrak{j}}}$ and hence $r_{x}=r_{y}$. 
Definition 3.3. Let $\left(L^{X}, \mathfrak{J}\right)$ be an $L$-LH $L$-topological space. The magnitude $L$-set of $\left(L^{X}, \mathfrak{J}\right)$ is an $L$-set in $X$ denoted by $L-m_{X}^{\mathfrak{T}}$ and defined by

$$
L-m_{X}^{\mathfrak{I}}(x)=r_{x}, \quad \text { where } r_{x} \text { is the magnitude of }\left(L^{X}, \mathfrak{J}\right) \text { at } x .
$$

Proposition 3.4. Let $\left(L^{X}, \mathfrak{J}\right)$ be an L-LH L-topological space. Then its magnitude L-set $L-m_{X}^{\mathfrak{Y}}$ is an open $L$-set in $\left(L^{X}, \mathfrak{J}\right)$.

Proof. For each $x \in X,\left(L-m_{X}^{\mathfrak{Y}}(x)\right) \chi_{L-C_{x}^{\mathfrak{T}}} \in \mathfrak{J}$ and thus, $L-m_{X}^{\mathfrak{Y}}=\bigvee_{x \in X}\left(L-m_{X}^{\mathfrak{J}}(x)\right) \chi_{L-C_{x}^{\mathfrak{T}}}$ $\in \mathfrak{J}$.

Theorem 3.5. Let $\left(L^{X}, \mathfrak{J}\right)$ be an $L$-LH L-topological space at $x$ and let $r_{x}$ be the magnitude of $\left(L^{X}, \mathfrak{I}\right)$ at $x$. If $a \in L$ with $a<r_{x}$, then $\left(X, \mathfrak{I}_{a}\right)$ is LH at $x$.

Proof. Let $a \in L$ with $a<r_{x}$. Since $r_{x} \chi_{L-C_{x}^{\mathfrak{I}}} \in \mathfrak{J},\left(r_{x} \chi_{L-C_{x}^{\mathfrak{I}}}\right)^{-1}(\downarrow a)=L-C_{x}^{\mathfrak{I}} \in \mathfrak{J}_{a}$. But by Proposition 1.17 , we have $L-C_{x}^{\mathfrak{J}} \subseteq C_{x}^{\mathfrak{J}_{a}}$. Therefore, $\left(X, \mathfrak{J}_{a}\right)$ is $L$-locally homogeneous at $x$.

Theorem 3.6. If $\left(L^{X}, \mathfrak{J}\right)$ is an $L$-LH L-topological space, then $\left(X, \mathfrak{J}_{0}\right)$ is $L H$.

Proof. Let $x \in X$ and let $L-m_{X}^{\mathfrak{I}}(x)=r_{x}$, then $r_{x} \chi_{L-C_{x}^{\mathfrak{I}}} \in \mathfrak{J}$, and $\left(r_{x} \chi_{C_{x}^{\mathfrak{I}}}\right)^{-1}(\downarrow 0)=L-C_{x}^{\mathfrak{I}} \in$ $\mathfrak{I}_{0}$. But by Proposition 1.17, we have $L-C_{x}^{\mathfrak{J}} \subseteq C_{x}^{\mathfrak{I}_{0}}$. Therefore, $\left(X, \mathfrak{I}_{0}\right)$ is LH at $x$ and hence $\left(X, \mathfrak{J}_{0}\right)$ is an LH topological space.

Example 3.7. Take $X=\mathbb{N}$ (the set of natural numbers), $L=I$, and $\mathfrak{I}$ the $L$-topology on $X$ generated by

$$
\boldsymbol{\beta}=\left\{1, p_{n}: p_{n} \text { is an } L \text {-point with support } n \text { and level } \frac{1}{n}, n \in \mathbb{N}\right\}
$$

as a base. It is not difficult to see that $\mathfrak{J}_{0}=\tau_{\text {disc }}$ and for each $n \in \mathbb{N}, L-C_{n}^{\mathfrak{J}}=\{n\}$. Therefore, by part (6) of Corollary 2.4, it follows that $\left(L^{X}, \mathfrak{J}\right)$ is an $L$-LH $L$-topological space. Now for each $n \in \mathbb{N}$, it is clear that $L-m_{X}^{\mathfrak{I}}(n)=1 / n$. If $n \in \mathbb{N}-\{1\}$ and $1 / n \leq a<1$, then $C_{n}^{\mathfrak{I}_{a}}=\{n, n+1, n+2, \ldots\} \notin \mathfrak{J}_{a}$ and so by Proposition 1.22 , it follows that $\left(X, \mathfrak{J}_{a}\right)$ is not LH at $n$. This shows that in Theorem 3.5, the condition " $a \in L$ with $a<r_{x}$ " cannot be dropped. On the other hand, if $0<a<1$, then there exists $n_{\circ} \in \mathbb{N}-\{1\}$ such that $1 / n_{\circ}<a$ and so $\left(X, \mathfrak{J}_{a}\right)$ is not $\mathrm{LH}$ at $n_{\mathrm{o}}$. This shows that the result in Theorem 3.6 cannot be replaced by " $\left(X, \mathfrak{J}_{a}\right)$ ” if $a \in L-\{0,1\}$.

THeOREm 3.8. There exists an L-topological space $\left(L^{X}, \mathfrak{J}\right)$ such that for each $a \in L-\{1\}$, $\left(X, \mathfrak{J}_{a}\right)$ is both homogeneous and $S L H$, but $\left(L^{X}, \mathfrak{I}\right)$ is not $L-L H$.

Proof. Let $X$ be an infinite set or a nonempty finite set with even cardinality, $L=I$. Choose $A \subseteq X$ such that $\operatorname{Card} A=\operatorname{Card}(X-A)$ and let

$$
\mathfrak{J}=\left\{0_{X}, 0.3 \chi_{A} \cup \chi_{X-A}, \chi_{A} \cup 0.3 \chi_{X-A}, 0.5 \chi_{A} \cup 0.3 \chi_{X-A}, 0.5 \chi_{A} \cup \chi_{X-A}, 0.3_{X}, 1_{X}\right\}
$$


Then $\mathfrak{J}$ is an $L$-topology on $X$. Let $a \in L-\{1\}$, then $\mathfrak{J}_{a}=\left\{\varnothing, X, A, A^{c}\right\}$ if $a \geq 0.3$ and $\mathfrak{J}_{a}=\{\varnothing, X\}$ if $a<0.3$. It is not difficult to see that $\left(X, \mathfrak{J}_{a}\right)$ is both homogeneous and SLH. Pick $x_{1} \in A$ and $x_{2} \in A^{C}$, then $L-C_{x_{1}}^{\mathfrak{J}}=A$ and $L-C_{x_{2}}^{\mathfrak{J}}=A^{c}$. Therefore, by part (6) of Corollary 2.4, it follows that $\left(L^{X}, \mathfrak{J}\right)$ is not $L$-LH.

Theorem 3.8 also shows that the converse of Theorem 3.6 is not true in general.

Example 3.7 shows that in $L$-LH $L$-topological spaces the magnitude $L$-set may not be a constant $L$-set. This leads us to the following two definitions.

Definition 3.9. An $L$-topological space $\left(L^{X}, \mathfrak{J}\right)$ is called $L$-CLH ( $L$-constant locally homogeneous) if it is $L$-LH and its magnitude $L$-set is a constant $L$-set.

Definition 3.10. An $L$-topological space $\left(L^{X}, \mathfrak{I}\right)$ is called $L$-ACLH ( $L$-absolutely constant locally homogeneous) if it is $L$-LH and its magnitude $L$-set $L-m_{X}^{\mathfrak{Y}}=1_{X}$.

Remark 3.11. Every $L$-CLH $L$-topological space is $L$-LH but the converse may not be true as Example 3.7 shows.

Remark 3.12. Every $L$-ACLH $L$-topological space is $L$-CLH but the converse may not be true.

Example 3.13. Let $X=\left\{x_{1}, x_{2}, x_{3}\right\}$, let $L=I$, and $\lambda, \mu$ are $L$-sets defined as follows:

$$
\begin{aligned}
\lambda\left(x_{1}\right)=0.5, & \lambda\left(x_{2}\right)=0, & \lambda\left(x_{3}\right)=0, \\
\mu\left(x_{1}\right)=0, & \mu\left(x_{2}\right)=0.5, & \mu\left(x_{3}\right)=0.5 .
\end{aligned}
$$

Let $\mathfrak{J}=\left\{0_{X}, \lambda, \mu, 0.5_{X}, 1_{X}\right\}$. It is not difficult to see that $L-C_{x_{1}}^{\mathfrak{I}}=\left\{x_{1}\right\}, L-C_{x_{2}}^{\mathfrak{J}}=\left\{x_{2}\right.$, $\left.x_{3}\right\}$, and $\mathfrak{J}_{0}=\left\{\varnothing,\left\{x_{1}\right\},\left\{x_{2}, x_{3}\right\}\right\}$. Thus, by part (6) of Corollary 2.4, it follows that $\left(L^{X}, \mathfrak{J}\right)$ is an $L$-LH $L$-topological space. On the other hand, it is clear that $L-m_{X}^{\mathfrak{Y}}=0.5_{X}$. Therefore, the $L$-topological space $\left(L^{X}, \mathfrak{I}\right)$ is $L$-CLH but not $L$-ACLH.

Example 3.13 also shows that the converse of Theorem 3.5 is not true in general.

Theorem 3.14. Let $(X, \tau)$ be a topological space and let $L$ be a fuzzy lattice. Then the following are equivalent:

(1) $\left(L^{X}, \omega_{L}(\tau)\right)$ is $L-A C L H$,

(2) $\left(L^{X}, \omega_{L}(\tau)\right)$ is $L-C L H$,

(3) $(X, \tau) L H$.

Proof. $(1) \Rightarrow(2)$. Obvious.

$(2) \Rightarrow(3)$. Corollary 2.6.

$(3) \Rightarrow(1)$. If $(X, \tau)$ is LH topological space, then by Corollary 2.6, it follows that $\left(L^{X}\right.$, $\left.\omega_{L}(\tau)\right)$ is an $L$-LH $L$-topological space. On the other hand, if $x \in X$, then by Proposition 1.22 , it follows that $C_{x}^{\tau}$ is open in $(X, \tau)$. Therefore, by Proposition 1.14, it follows that $L-C_{x}^{\omega_{L}(\tau)}$ is open in $(X, \tau)$ and hence $\chi_{L-C_{x}^{\omega_{L}(\tau)}} \in \omega_{L}(\tau)$. Since $S\left(\chi_{L-C_{x}^{\omega_{L}(\tau)}}\right)=L-C_{x}^{\omega_{L}(\tau)} \subseteq$ $L-C_{x}^{\omega_{L}(\tau)}$, then $L-m_{X}^{\omega(\tau)}(x)=1$.

Corollary 3.15. The L-CLH property in L-topological spaces is a "good extension" of LH property in ordinary topological spaces. 
Corollary 3.16. The L-ACLH property in L-topological spaces is a "good extension" of LH property in ordinary topological spaces.

The following two results characterize, respectively, $L$-CLH and $L$-ACLH $L$-topological spaces.

Theorem 3.17. Let $\left(L^{X}, \mathfrak{I}\right)$ be an L-topological space. Then the following are equivalent.

(1) $\left(L^{X}, \mathfrak{J}\right)$ is $L-C L H$.

(2) There exists $c \in L-\{0\}$ such that for each $x \in X, c \chi_{L-C_{x}^{\mathfrak{J}}} \in \mathfrak{J}$ and $a \chi_{L-C_{x}^{\mathfrak{J}}} \notin \mathfrak{J}$ for every $a \in L$ with $a>c$.

Proof. (1) $\Rightarrow(2)$. By (1), it follows that there exists $c \in L-\{0\}$ such that $L-m_{X}^{\mathfrak{Y}}=c_{X}$. Then by part (4) of Corollary 2.4, it follows that $c \chi_{L-C_{x}^{\mathfrak{J}}} \in \mathfrak{J}$ for each $x \in X$ and for every $a \in L$ with $a>c, a \chi_{L-C_{x}^{\mathfrak{I}}} \notin \mathfrak{J}$.

$(2) \Rightarrow(1)$. By $(2)$, and part (5) of Corollary 2.4 , it follows that $\left(L^{X}, \mathfrak{I}\right)$ is $L$-LH. Thus by part (4) of Corollary 2.4 and (2), it follows that $\bigvee\left\{\lambda \in \mathfrak{J}: S(\lambda) \subseteq L-C_{x}^{\mathfrak{J}}\right\}=c \chi_{L-C_{x}^{\mathfrak{J}}}$ at each $x \in X$. Hence $L-m_{X}^{\mathfrak{Y}}=c_{X}$.

Theorem 3.18. Let $\left(L^{X}, \mathfrak{J}\right)$ be an $L$-topological space. Then the following are equivalent.

(1) $\left(L^{X}, \mathfrak{I}\right)$ is an $L-A C L H$.

(2) For each $x \in X, \chi_{L-C_{x}^{\mathfrak{x}}} \in \mathfrak{J}$.

(3) The L-homogeneous components $L-C_{x}^{\mathfrak{J}}$ of $\left(L^{X}, \mathfrak{J}\right)$ are clopen subspaces of $\left(L^{X}, \mathfrak{J}\right)$.

Proof. (1) $\Rightarrow(2)$. By (1), it follows that $L-m_{X}^{\mathfrak{Y}}=1_{X}$. Then by part (4) of Corollary 2.4 , it follows that $\chi_{L-C_{x}^{\mathfrak{g}}} \in \mathfrak{J}$ for each $x \in X$.

$(2) \Rightarrow(3)$. Let $x \in X$. Then by (2), $L-C_{x}^{\mathfrak{I}}$ is an open subspace of $\left(L^{X}, \mathfrak{J}\right)$. On the other hand, it is not difficult to see that $\left(\chi_{L-C_{x}^{\mathfrak{y}}}\right)^{\prime}=\bigvee\left\{\chi_{L-C_{y}^{\mathfrak{y}}}: y \notin L-C_{x}^{\mathfrak{J}}\right\}$. Therefore, $\left(\chi_{L-C_{x}^{\mathfrak{y}}}\right)^{\prime} \in$ $\mathfrak{J}$ and hence $L-C_{x}^{\mathfrak{J}}$ is a closed subspace of $\left(L^{X}, \mathfrak{I}\right)$.

(3) $\Rightarrow(1)$. By (3), and part (5) of Corollary 2.4, it follows that $\left(L^{X}, \mathfrak{J}\right)$ is $L$-LH. Thus by part (4) of Corollary 2.4 and (3), it follows that $\bigvee\left\{\lambda \in \mathfrak{J}: S(\lambda) \subseteq L-C_{x}^{\mathfrak{J}}\right\}=\chi_{L-C_{x}^{\mathfrak{J}}}$ at each $x \in X$. Hence $L-m_{X}^{\mathfrak{J}}=1_{X}$.

Theorem 3.19. Every L-SLH L-topological space is L-ACLH.

Proof. Let $\left(L^{X}, \mathfrak{I}\right)$ be an $L$-SLH $L$-topological space. Then by Theorem 2.8 , it follows that $\left(L^{X}, \mathfrak{J}\right)$ is $L$-LH. If $\left(L^{X}, \mathfrak{I}\right)$ is not $L$-ACLH, then there exists $y \in X$ such that $L-m_{X}^{\mathfrak{I}}(y)=$ $r<1$. Since $\left(L^{X}, \mathfrak{J}\right)$ is $L-S L H$, then there exists a representable base $\boldsymbol{\beta}$ for $\mathfrak{I}$. Since $\bigvee \boldsymbol{\beta}=1$, then $(\bigvee \boldsymbol{\beta})(y)=1$ and so there exists $\lambda \in \mathfrak{J}$ such that $\lambda(y)>r$. Therefore, $y \in S(\lambda)$ and if $z \in S(\lambda)$, then there exists $h \in \operatorname{LHOM}\left(L^{X}, \mathfrak{J}\right)$ such that $h(y)=z$ and $h(t)=t$ for all $t \in$ $X-S(\lambda)$. Thus, $S(\lambda) \subseteq L-C_{y}^{\mathfrak{I}}$ and hence $\lambda(y) \leq L-m_{X}^{\mathfrak{J}}(y)$ which is absurd. Therefore, $\left(L^{X}, \mathfrak{J}\right)$ is $L$-ACLH.

The following result is a consequence of Theorems 3.18 and 3.19.

Corollary 3.20. If $\left(L^{X}, \mathfrak{I}\right)$ is L-SLH L-topological space, then every L-homogeneous component $L-C_{x}^{\mathfrak{J}}$ of $\left(L^{X}, \mathfrak{I}\right)$ is a clopen subspace of $\left(L^{X}, \mathfrak{J}\right)$.

Corollary 3.21 [3]. If $\left(I^{X}, \mathfrak{I}\right)$ is I-SLH I-topological space, then every I-homogeneous component $I-C_{x}^{\mathfrak{J}}$ of $\left(I^{X}, \mathfrak{I}\right)$ is a clopen subspace of $\left(I^{X}, \mathfrak{I}\right)$. 
Remark 3.22. The converse of Theorem 3.19 is not true in general. This follows from Theorem 3.14 and Propositions 1.20 and 1.24.

Theorem 3.23. Let $\left(L^{X}, \mathfrak{J}\right)$ be an L-topological space. Then the following are equivalent.

(1) $\left(L^{X}, \mathfrak{I}\right)$ is an $L$-SLH L-topological space.

(2) $\left(L^{X}, \mathfrak{J}\right)$ is an $L$-ACLH L-topological space and for each $x \in X$ the L-topological subspace $\left(L-C_{x}^{\mathfrak{J}}, \mathfrak{J}_{L-C}\right)$ is $L-S L H$.

(3) The L-homogeneous components of $\left(L^{X}, \mathfrak{I}\right)$ are clopen and L-SLH subspaces of $\left(L^{X}, \mathfrak{J}\right)$.

Proof. $(1) \Rightarrow(2)$. Since $\left(L^{X}, \mathfrak{J}\right)$ is $L$-SLH, then by Theorem 3.19 , it follows that $\left(L^{X}, \mathfrak{J}\right)$ is $L$-ACLH. Let $x \in X$. Thus by Theorem 3.18, it follows that $\chi_{L-C_{x}^{\mathfrak{F}}} \in \mathfrak{J}$. Let $\beta$ be a representable base for $\mathfrak{I}$ and let $\boldsymbol{\beta}_{x}=\left\{\lambda_{\mid L-C_{x}^{\mathfrak{J}}}: \lambda \in \boldsymbol{\beta}\right\}$. It is not difficult to see that $\boldsymbol{\beta}_{x}$ is a base for $\mathfrak{J}_{L-C_{x}^{\mathfrak{y}}}$. To see that $\boldsymbol{\beta}_{x}$ is representable, let $y, z \in S\left(\lambda_{\mid L-C_{x}^{\mathfrak{g}}}\right)=S(\lambda) \cap L-C_{x}^{\mathfrak{J}}$. Since $\boldsymbol{\beta}$ is a representable base for $\mathfrak{I}$, then there exists $h \in \operatorname{LHOM}\left(L^{X}, \mathfrak{I}\right)$ such that $h(y)=z$ and $h(t)=t$ for all $t \in X-S(\lambda)$. Now by Proposition 1.12, it follows that $h\left(L-C_{x}^{\mathfrak{J}}\right)=L-C_{x}^{\mathfrak{J}}$. Moreover, it is clear that $h(t)=t$ for all $t \in L-C_{x}^{\mathfrak{J}}-S\left(\lambda_{\mid L-C_{x}^{\mathfrak{J}}}\right)$. To complete the proof of this direction, it is sufficient to show that the restriction $h_{\mid L-C_{x}^{\mathfrak{J}}}:\left(L-C_{x}^{\mathfrak{J}}, \mathfrak{J}_{L-C_{x}^{\mathfrak{J}}}\right) \rightarrow(L-$ $\left.C_{x}^{\mathfrak{I}}, \mathfrak{J}_{L-C_{x}^{\mathfrak{I}}}\right)$ of $h$ on $L-C_{x}^{\mathfrak{J}}$ is an $L$-homeomorphism. In fact, for each $\lambda \in \mathfrak{J}, h^{\leftarrow}\left(\lambda_{\mid L-C_{x}^{\mathfrak{I}}}\right)=$ $\left(h^{-}(\lambda)\right)_{\mid L-C_{x}^{\mathfrak{J}}}$. This shows that $h_{\mid L-C_{x}^{\mathfrak{J}}}:\left(L-C_{x}^{\mathfrak{J}}, \mathfrak{J}_{L-C_{x}^{\mathfrak{I}}}\right) \rightarrow\left(L-C_{x}^{\mathfrak{J}}, \mathfrak{J}_{L-C_{x}^{\mathfrak{J}}}\right)$ is an $L$-continuous function. Similarly, we can show that $\left(h_{\mid L-C_{x}^{\mathfrak{g}}}\right)^{-1}:\left(L-C_{x}^{\mathfrak{g}}, \mathfrak{I}_{L-C_{x}^{\mathfrak{g}}}\right) \rightarrow\left(L-C_{x}^{\mathfrak{J}}, \mathfrak{I}_{L-C_{x}^{\mathfrak{g}}}\right)$ is $L$-continuous. Finally, it is clear that $h_{\mid L-C_{x}^{\mathfrak{j}}}:\left(L-C_{x}^{\mathfrak{J}}, \mathfrak{J}_{L-C_{x}^{\mathfrak{I}}}\right) \rightarrow\left(L-C_{x}^{\mathfrak{J}}, \mathfrak{J}_{L-C_{x}^{\mathfrak{J}}}\right)$ is a bijection.

(2) $\Rightarrow(3)$. Follows from Theorem 3.18.

$(3) \Rightarrow(1)$. For each $x \in X$, choose a representable base $\boldsymbol{\beta}_{x}$ for $\left(L-C_{x}^{\mathfrak{J}}, \mathfrak{J}_{L-C_{x}^{\mathfrak{J}}}\right)$. For every $x \in X$ and $\lambda \in \boldsymbol{\beta}_{x}$, choose $\lambda^{*} \in \mathfrak{I}$ such that $\lambda=\lambda_{\mid L-C_{x}^{\mathfrak{g}}}^{*}$. For every $x \in X$ let $\boldsymbol{\beta}_{x}^{*}=\left\{\lambda^{*} \wedge\right.$ $\left.\chi_{L-C_{x}^{\mathfrak{x}}}: \lambda \in \boldsymbol{\beta}_{x}\right\}$. Let $\boldsymbol{\beta}^{*}=\cup\left\{\boldsymbol{\beta}_{x}^{*}: x \in X\right\}$.

Claim 3.24. $\boldsymbol{\beta}^{*}$ is a representable base for $\mathfrak{T}$.

Proof of claim. Since each component is a clopen subspace of $\left(L^{X}, \mathfrak{J}\right)$, it follows that $\beta^{*} \subseteq$ $\mathfrak{I}$. Let $\mu \in \mathfrak{I}$ with $\mu \neq 0_{X}$. Then for each $x \in X, \mu_{\mid L-C_{x}^{\mathfrak{J}}} \in \mathfrak{I}_{L-C_{x}^{\mathfrak{J}}}$ and so there exists $\widetilde{\mathbf{B}}_{x} \subseteq \boldsymbol{\beta}_{x}$ such that $\mu_{\mid L-C_{x}^{\mathfrak{I}}}=\bigvee \widetilde{\mathbf{B}}_{x}$. For each $x \in X$, it is easy to see that $\mu \wedge \chi_{L-C_{x}^{\mathfrak{y}}}=\bigvee\left\{\lambda^{*} \wedge \chi_{L-C_{x}^{\mathfrak{g}}}\right.$ : $\left.\lambda \in \widetilde{\mathbf{B}}_{x}\right\}$. Therefore, since $\mu=\mu \wedge 1_{X}=\mu \wedge\left(\bigvee\left\{\chi_{L-C_{x}^{\mathfrak{J}}}: x \in X\right\}\right)=\bigvee\left\{\mu \wedge \chi_{L-C_{x}^{\mathfrak{J}}}: x \in X\right\}$, it follows that $\mu$ is a join of members of $\boldsymbol{\beta}^{*}$. Hence $\boldsymbol{\beta}^{*}$ is a base for $\mathfrak{J}$. To show that $\boldsymbol{\beta}^{*}$ is representable, let $\lambda^{*} \wedge \chi_{L-C_{x}^{\mathfrak{g}}} \in \boldsymbol{\beta}^{*}$ where $x \in X$ and $\lambda \in \boldsymbol{\beta}_{x}$ and let $y, z \in S\left(\lambda^{*} \wedge \chi_{L-C_{x}^{\mathfrak{g}}}\right)=$ $S(\lambda) \cap L-C_{x}^{\mathfrak{J}}$. Since $\boldsymbol{\beta}_{x}$ is a representable base for $\mathfrak{J}_{L-C_{x}^{\mathfrak{g}}}$, there exists $f \in \operatorname{LHOM}(L-$ $\left.C_{x}^{\mathfrak{J}}, \mathfrak{J}_{L-C_{x}^{\mathfrak{I}}}\right)$ such that $f(y)=z$ and $f(t)=t$ for all $t \in L-C_{x}^{\mathfrak{J}}-S(\lambda)$. Define $h:\left(L^{X}, \mathfrak{J}\right) \rightarrow$ $\left(L^{X}, \mathfrak{J}\right)$ by

$$
h(t)= \begin{cases}f(t) & \text { if } t \in L-C_{x}^{\mathfrak{J}}, \\ t & \text { if } t \in X-L-C_{x}^{\mathfrak{J}} .\end{cases}
$$

Let $\mu^{*} \wedge \chi_{L-C_{y}^{\mathfrak{s}}} \in \boldsymbol{\beta}^{*}$ where $y \in X$ and $\mu \in \boldsymbol{\beta}_{y}$. We have two cases on $y$. 
Case 1. $L-C_{x}^{\mathfrak{J}}=L-C_{y}^{\mathfrak{I}}$, in this case $\mu \in \boldsymbol{\beta}_{x}$ and so $f^{-}(\mu) \in \mathfrak{J}_{L-C_{x}^{\mathfrak{I}}}$. Thus there exists $\delta \in \mathfrak{I}$ such that $\delta_{\mid L-C_{x}^{\mathfrak{J}}}=f^{\leftarrow}(\mu)$ and hence $h^{\leftarrow}\left(\mu^{*} \wedge \chi_{L-C_{y}^{\mathfrak{I}}}\right)=\delta \wedge \chi_{L-C_{x}^{\mathfrak{I}}} \in \mathfrak{J}$.

Case 2. $L-C_{x}^{\mathfrak{J}} \cap L-C_{y}^{\mathfrak{J}}=\varnothing$, in this case $h^{\leftarrow}(\mu)=\mu$.

Therefore, $h$ is an $L$-continuous function. Similarly we can show that $h^{-1}$ is $L$-continuous. Since $h$ is obviously bijective, it follows that $h \in \operatorname{LHOM}\left(L^{X}, \mathfrak{I}\right)$. Now it is clear that $h(y)=z$ and $h(t)=t$ for all $t \in X-S\left(\lambda^{*} \wedge \chi_{L-C_{x}^{\mathfrak{y}}}\right)$. Therefore, $\boldsymbol{\beta}^{*}$ is a representable base for $\mathfrak{I}$ as we claimed.

The above claim shows that $\left(L^{X}, \mathfrak{I}\right)$ is an $L$-SLH $L$-topological space as required.

Theorem 3.25. Every L-homogeneous L-topological space is L-ACLH.

Proof. Let $\left(L^{X}, \mathfrak{J}\right)$ be an $L$-homogeneous $L$-topological space. Then for each $x \in X, L-$ $C_{x}^{\mathfrak{J}}=X$ and so it follows that $\left(L-C_{x}^{\mathfrak{J}}, \mathfrak{J}_{L-C_{x}^{\mathfrak{I}}}\right)$ is a clopen subspace of $\left(L^{X}, \mathfrak{J}\right)$. Therefore, by Theorem 3.18, it follows that $\left(L^{X}, \mathfrak{J}\right)$ is $L$-ACLH.

Remark 3.26. The converse of Theorem 3.25 is not true in general. This follows from Theorem 3.14 and Propositions 1.21 and 1.25.

Remark 3.27. Theorems 3.19, 3.25 and Remarks 3.11, 3.12 reveal that the following diagram of implications is true for $L$-topological spaces:

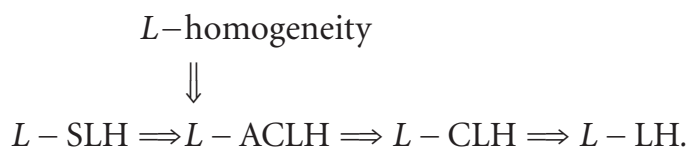

In Theorem 3.6, if the condition $L-\mathrm{LH}$ is replaced by the condition $L-C L H$, then the conclusion will be better as the following result shows.

Theorem 3.28. Let $\left(L^{X}, \mathfrak{J}\right)$ be an L-CLH L-topological space with magnitude $L$-set $L-$ $m_{X}^{\mathfrak{I}}=c_{X}$. If $a \in L$ with $a<c$, then $\left(X, \mathfrak{J}_{a}\right)$ is $L H$.

Proof. Let $a \in L$ with $a<c$ and let $x \in X$. Then $c \chi_{L-C_{x}^{\mathfrak{g}}} \in \mathfrak{J}$ and $\left(c \chi_{L-C_{x}^{\mathfrak{g}}}\right)^{-1}(\downarrow a)=L-$ $C_{x}^{\mathfrak{J}} \in \mathfrak{J}_{a}$. Therefore, by Proposition 1.17, it follows that $x \in L-C_{x}^{\mathfrak{J}} \subseteq C_{x}^{\mathfrak{J}_{a}}$ and hence $\left(X, \mathfrak{J}_{a}\right)$ is LH at $x$. Since $x$ is arbitrary, the proof is complete.

Corollary 3.29. If $\left(L^{X}, \mathfrak{J}\right)$ is an $L$-ACLH L-topological space, then for each $a \in L-\{0\}$, $\left(X, \mathfrak{J}_{a}\right)$ is $L H$.

Remark 3.30. Example 3.13 shows that the converse of Theorem 3.28 is not true in general.

Remark 3.31. Theorem 3.8 shows that the converse of Corollary 3.29 is not true in general.

The following example shows that the condition " $a \in L$ with $a<c$ " is necessary in order for Theorem 3.28 to hold. 
Example 3.32. Let $X=\left\{x_{1}, x_{2}\right\} L=I$, and $\lambda, \mu$, and $v$ are $L$-sets defined as follows:

$$
\begin{array}{ll}
\lambda\left(x_{1}\right)=0.5, & \lambda\left(x_{2}\right)=0, \\
\mu\left(x_{1}\right)=0, & \mu\left(x_{2}\right)=0.5, \\
v\left(x_{1}\right)=0.5, & \nu\left(x_{2}\right)=1 .
\end{array}
$$

Let $\mathfrak{J}=\left\{0_{X}, \lambda, \mu, v, 0.5_{X}, 1_{X}\right\}$. It is not difficult to see that $L-C_{x_{1}}^{\mathfrak{I}}=\left\{x_{1}\right\}, L-C_{x_{2}}^{\mathfrak{I}}=$ $\left\{x_{2}\right\}, \mathfrak{J}_{0}=\tau_{\text {disc }}$, and $L-m_{X}^{\mathfrak{I}}=0.5_{X}$. Thus, by part (6) of Corollary 2.4, it follows that $\left(L^{X}, \mathfrak{J}\right)$ is an $L$-LH $L$-topological space. On the other hand, for each $a \geq 0.5, \mathfrak{J}_{a}=\{\varnothing, X$, $\left.\left\{x_{2}\right\}\right\}$ and so $\left(L^{X}, \mathfrak{J}_{a}\right)$ is not LH topological space.

\section{References}

[1] S. Al Ghour, Homogeneous L-topological spaces, Master's thesis, Department of Mathematics, Yarmouk University, Irbid-Jordan, 1995.

[2] Homogeneity in fuzzy spaces and their induced spaces, Questions and Answers in General Topology 21 (2003), no. 2, 185-195.

[3] _ SLH fuzzy spaces, African Diaspora Journal of Mathematics 2 (2004), no. 2, 61-67.

[4] C. L. Chang, Fuzzy topological spaces, Journal of Mathematical Analysis and Applications 24 (1968), no. 1, 182-190.

[5] B. Fitzpatrick Jr. and H. X. Zhou, A survey of some homogeneity properties in topology, Papers on General Topology and Related Category Theory and Topological Algebra (New York, 1985/1987), Ann. New York Acad. Sci., vol. 552, New York Academy of Sciences, New York, 1989, pp. 28-35.

[6] A. A. Fora, New and old types of homogeneity, Turkish Journal of Mathematics 24 (2000), no. 4, 335-344.

[7] A. A. Fora and S. Al Ghour, Homogeneity in fuzzy spaces, Questions and Answers in General Topology 19 (2001), no. 2, 159-164.

[8] L. R. Ford Jr., Homeomorphism groups and coset spaces, Transactions of the American Mathematical Society 77 (1954), 490-497.

[9] J. A. Goguen, L-fuzzy sets, Journal of Mathematical Analysis and Applications 18 (1967), no. 1, $145-174$.

[10] U. Höhle and S. E. Rodabaugh (eds.), Mathematics of Fuzzy Sets. Logic, Topology, and Measure Theory, The Handbooks of Fuzzy Sets Series, vol. 3, Kluwer Academic, Massachusetts, 1999.

[11] Y.-M. Liu and M.-K. Luo, Fuzzy Topology, Advances in Fuzzy Systems-Applications and Theory, vol. 9, World Scientific, New Jersey, 1997, 1998 (English).

[12] W. Sierpenski, Sur uni propriété topologique des ensembles de'nombrable dense en soi, Fundamenta Mathematicae 1 (1920), 11-28.

[13] L. A. Zadeh, Fuzzy sets, Information and Control 8 (1965), no. 3, 338-353.

Samer Al Ghour: Department of Mathematics and Statistics, Jordan University of Science and Technology, Irbid 22110, Jordan

E-mail address: algore@just.edu.jo 


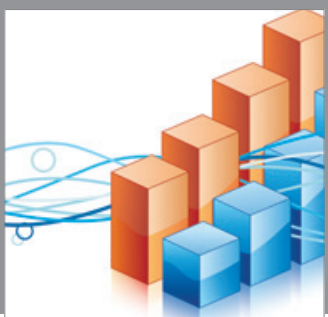

Advances in

Operations Research

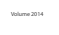

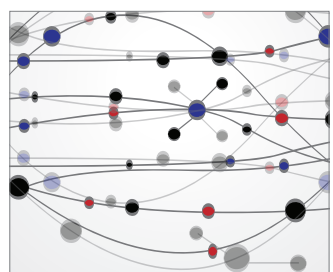

\section{The Scientific} World Journal
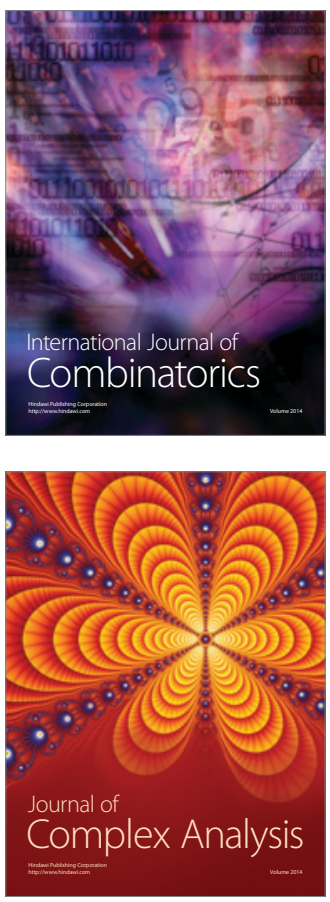

International Journal of

Mathematics and

Mathematical

Sciences
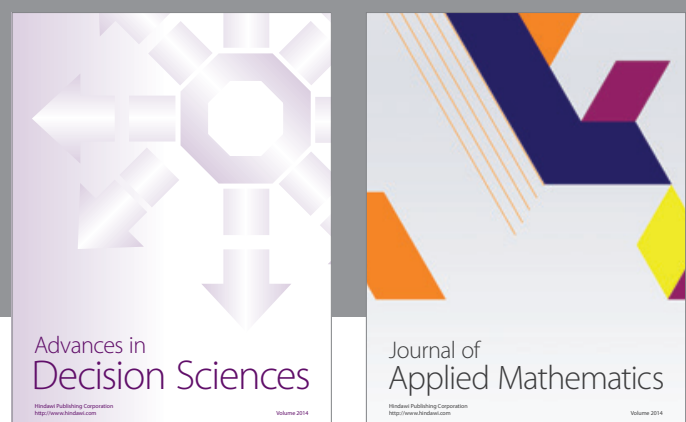

Journal of

Applied Mathematics
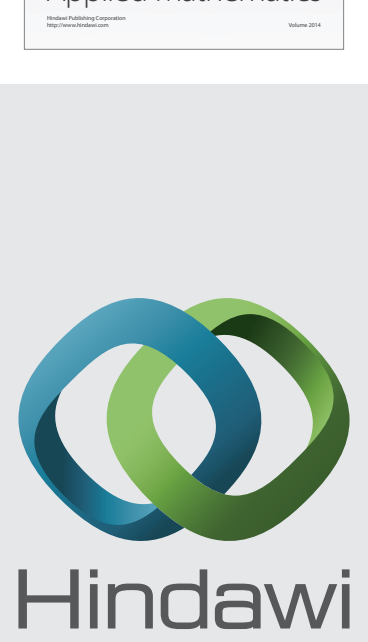

Submit your manuscripts at http://www.hindawi.com
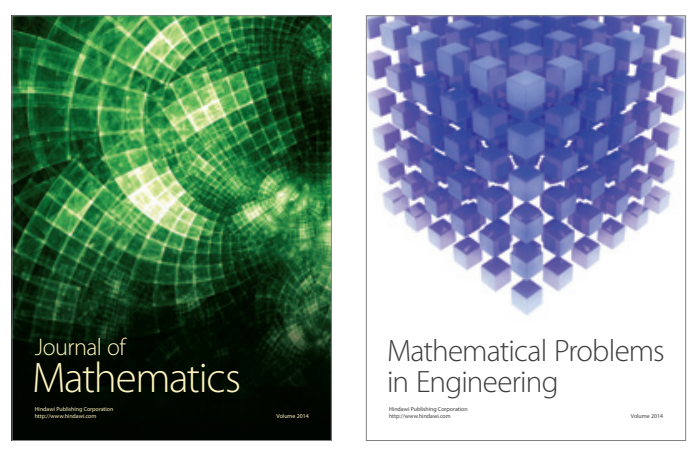

Mathematical Problems in Engineering
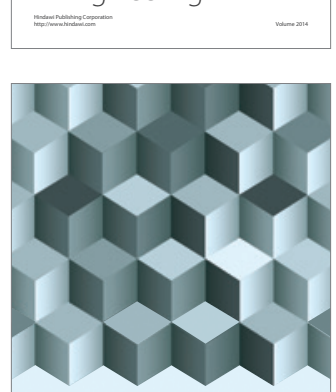

Journal of

Function Spaces
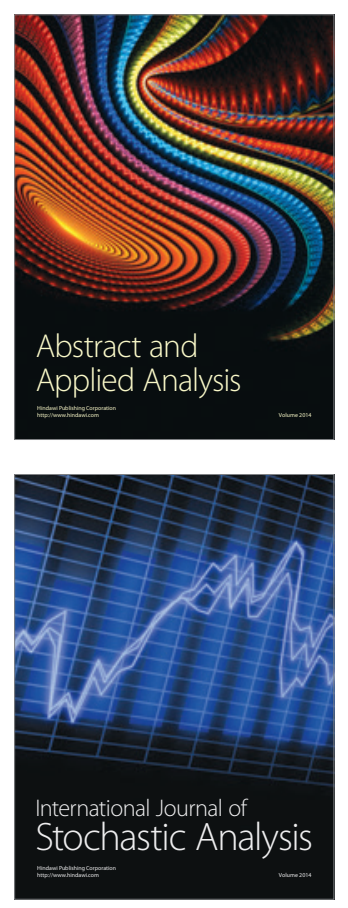

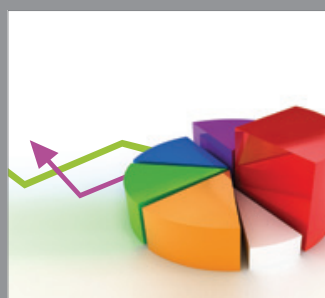

ournal of

Probability and Statistics

Promensencen
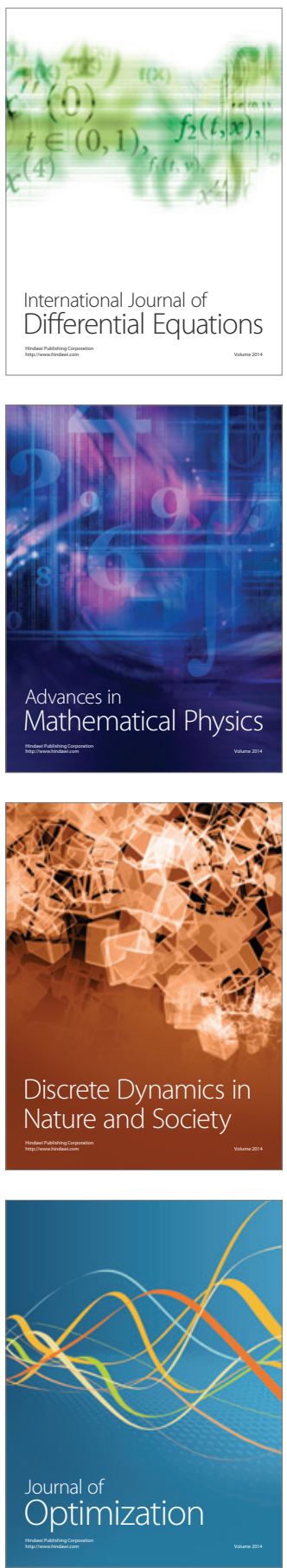